da Interculturalidade

à Tecnologia

\title{
Taxonomia dos tipos de tendências: uma proposta concisa
}

Amanda Queiroz Campos é Doutora em Design na modalidade cotutela (doutorado binacional) pela Universidade Federal de Santa Catarina (UFSC) e pela Bergische Universität Wuppertal (BUW), na Alemanha. Tem mestrado em Design e Expressão Gráfica (UFSC, 2013), graduação em Design pela mesma instituição (UFSC) e também em Moda com Habilitação em Estilismo pela Universidade do Estado de Santa Catarina (UDESC). $\mathrm{Na}$ UDESC, atua como professora de Moda nas áreas de Comunicação, Consumo

e Criação. Tem expressiva publicação científica nas áreas de Moda e Design em revistas nacionais e internacionais. Também atua como colaboradora e consultora em comportamento de consumo e em tendências. <amandaqc88@gmail.com> ORCID: 0000-0001-9291-2979
Resumo A palavra tendência teve uso extensivo e sofre associações forçosas, sendo usada como justificativa ou qualificação de produtos e serviços dos mais diversos fins. Além disso, aplica-se uma gama de taxonomias dentro do escopo semântico da palavra, o que compromete seu entendimento e estudo. O objetivo deste trabalho foi reduzir a complexidade sobre o conceito de tendência e normatizar suas terminologias. A pesquisa realizada teve natureza qualitativa e abordagem analítica. A metodologia decorreu das pesquisas bibliográfica e comparativa, relacionando dados de diferentes autores sobre as taxonomias que aplicavam para caracterizar diferentes tipologias de tendências. Aplicando a codificação axial (Grounded Theory), pode-se reduzir a quantidade de termos em dois: macrotendências e microtendências. Macrotendências correspondem a tendências socioculturais amplas, tendo longa duração e alto impacto. Microtendências são menos duradoras e impactantes, atingem apenas alguns segmentos do mercado ao apresentar orientações para produtos e serviços.

Palavras chave Tendências, Estudo de Tendências, Taxonomia. 


\section{Taxonomy of trends' types: a concise proposal}

Abstract The word trend was extensively used, and forcedly associated with products and services as an argument, qualification, or legitimation. In addition, one sees the application of a range of various taxonomies within the semantic scope of the word, which compromises their understanding and study. The objective of this study was to reduce the complexity of the concept and to standardize its terminologies. The nature of the research was qualitative and analytical. The methodology approach comprised bibliographical and comparative research, contrasting data from different authors regarding the taxonomies applied to characterize different typologies of trends. Applying axial coding (Grounded Theory), the quantity of terms was reduced to two: macro trends and micro trends. Macro trends correspond to broad sociocultural tendencies that have long duration and high impact. Micro trends are less durable and impactful, reaching only a few market segments by presenting guidelines for products and services.

Keywords Trends, Trend Studies, Taxonomy..

\section{Taxonomía de los tipos de tendencias: una propuesta concisa}

Resumen La palabra tendencia se ha utilizado ampliamente y tiene fuertes asociaciones, siendo utilizada como justificación o calificación de productos y servicios de los más diversos fines. Además, se aplica una gama de taxonomías dentro del alcance semántico de la palabra, lo que compromete su comprensión y estudio. El objetivo de este trabajo fue reducir la complejidad del concepto de tendencia y estandarizar sus terminologías. La investigación realizada tuvo un carácter cualitativo y un enfoque analítico. La metodología resultó de la investigación bibliográfica y comparativa, relacionando datos de diferentes autores sobre las taxonomías que aplicaron para caracterizar diferentes tipos de tendencias. Aplicando la codificación axial (teoría fundamentada), se puede reducir el número de términos en dos: macro tendencias y micro tendencias. Las macro tendencias corresponden a tendencias socioculturales amplias, de larga duración y alto impacto. Las micro tendencias son menos duraderas e impactantes, llegando solo a algunos segmentos del mercado al presentar pautas para productos y servicios.

Palabras clave Tendencias, Estudio de Tendencias, Taxonomía. 


\section{Introdução}

As tendências dão ênfase às mudanças e sua pesquisa consiste em mapear o contexto sociocultural para oferecer diretrizes às empresas para que delineiem planos de ação fundamentados no futuro. Segundo especialistas de gestão, a determinação de estratégias empresariais não deve ser uma ação intuitiva; requer planejamento e antecedência para que as ações sejam efetivas (PALADINI, 2009). Nos estudos e negócios do design - com ênfase no design de moda -, há o interesse pela prospecção de tendências que antecipam as influências no comportamento e as preferências da sociedade de modo geral.

Profissionais de design e marketing investem quantias consideráveis todos os anos para manter as marcas sintonizadas com as tendências, usando-as como artifícios para manutenção e expansão no mercado. Não surpreendentemente, as empresas especializadas à prospecção de tendências alcançam alto faturamento. As influências observadas, analisadas e editadas pelos pesquisadores de tendências operam como uma espécie de "espelho do futuro do mercado". Elas correspondem a expressão do espírito do tempo (Zeitgeist) na visualidade e materialidade de produtos.

Coloquialmente, tendência é associada às ideias de moda e à novidade. De acordo com o dicionário, a palavra tem o significado de inclinação, propensão, disposição, intenção, movimento e força determinante (BUARQUE-DE-HOLANDA, 1999). Pode-se afirmar que o termo tendência envolve vetores que indicariam a direção a seguir no futuro próximo ou distante (CAMPOS, 2017). De modo semelhante ao que ocorre com a palavra design, a palavra tendência foi aplicada extensivamente e sofreu associações forçosas. Além do uso extensivo, vê-se a aplicação de uma ampla gama de taxonomias associadas ao significado do termo tendência, entre elas: trend, onda, moda, fad, hype, megatendência, tendência de curto prazo, tendência de longo prazo, tendência de produto etc.

\section{Objetivos}

Este texto consiste em substrato da tese de doutorado intitulada of tradition and creation: the discourse of fashion designers on trend report usage desenvolvida em modalidade cotutela junto ao Programa de Pós-Graduação em Design da Universidade Federal de Santa Catarina (Pós-Design/UFSC) e do Departamento de Artes e Design da Universidade de Wuppertal (Alemanha). De modo geral, a pesquisa abordou o usufruto da informação de tendências de moda por designers de empresas consagradas. Todavia, o recorte selecionado e aprofundado neste artigo objetivou promover a redução de complexidade sobre o conceito e, também, normatizar suas terminologias. Sendo assim, sua principal contribuição é propor a manutenção de apenas duas categorias: macrotendências e microtendências - manutenção fundamentada na pesquisa e interpretação da bibliografia desenvolvidas e que serão apresentadas nas seguintes páginas. 


\section{Metodologia}

A pesquisa realizada tem natureza qualitativa e abordagem analítica, sua metodologia decorre da combinação da pesquisa bibliográfica com a comparativa. A abordagem específica de comparação consiste no contraste entre diferentes autores consagrados nos estudos das tendências sobre as taxonomias que aplicavam para caracterizar diferentes tipologias de tendências. Considerou-se as correntes teóricas quanto às suas terminologias adotadas, similaridades e diferenças. A listagem de termos tipológicos levantou, por sua vez, a urgência da explicitação de quais parâmetros de classificação haviam sido considerados para a determinação das diferenças entre as categorias - o que não é explicitado pela maior parte do corpus teórico.

o esclarecimento quanto aos parâmetros comparativos possibilitou estabelecer relações entre os vários conceitos listados pela bibliografia. De forma a reduzir a quantidade de termos, aplicou-se a codificação axial segundo postula a Grounded Theory (CORBIN; STRAUSS, 1990; SONEIRA, 2007). Buscou-se organizar de forma sistemática a ampla gama de termos ao redor de dois termos centrais: macrotendências e microtendências. Por fim, desenvolveram-se esforços para conceituar ambos esses termos tendo como diretrizes os parâmetros de diferenciação também emergentes da análise e interpretação dos dados bibliográficos.

\section{0 conceito de tendência}

Para proceder o estudo das taxonomias de tendências é evidentemente relevante que o termo tendência, em si, seja conceituado. Em relação à dinâmica do mercado, o conceito de tendência atualmente abarca novidades estéticas adotadas em produtos, servindo como ferramentas para coordenação entre oferta e demanda. A literatura histórica produzida sobre o fenômeno das mudanças constantes refere-se a um período em que as tendências ainda não foram institucionalizadas - muitas vezes usando a terminologia modas (LEHNERT, KÜHL e WEISE, 2014; SIMMEL, 2015; VEBLEN, 2013). Ao considerar as modas, os autores abordam as mudanças devido ao gosto pela mudança em si. Porém, tendências não são essencialmente tendências de moda ou envolvem interesse econômico (LIEBERSON, 2000).

A associação entre tendências e moda explica-se pela percepção do ritmo acelerado com que as mudanças ocorrem em roupas, acessórios e penteados. Consideradas estratégicas, tendências fazem referência a um contexto sociocultural, mesmo nos casos em que a explicação sociocultural da tendência se esconde por detrás do desejo pela mudança (SVENDSEN, 2010). As tendências consistem em transformações que envolvem as esferas social, cultural, individual e estética. Tais transformações são aceitas como positivas devido à lógica da ordenação, renovação e normalização da 
mudança fundamentada no ethos moda. As tendências podem ser mudanças autolegitimadas, mas elas alinham-se, em maior ou menor grau, com o espírito da época, Zeitgeist.

Por sua vez, as consideradas tendências da moda são expressões das tendências socioculturais em esferas estéticas, principalmente por meio de qualidades visuais e táteis aplicadas aos produtos de vestuário. A possibilidade de aplicar tendências em produtos deriva do esforço em prognosticá-las. Estudos de tendências, previsão de tendências e estudos do futuro são práticas de conjecturas de cenários futuros (PETERMANN, 2014; RECH e SILVEIRA, 2017). No campo do design, as tendências são utilizadas para melhorar o desenvolvimento, a inovação e a atração sensorial dos produtos, principalmente através da estética (BUCK, HERMANN e LUBKOWITZ, 1998).

Muitas empresas estão ativamente envolvidas com pesquisa e adoção de tendências, enquanto outras as percebem ceticamente. Se entendermos as tendências apenas como sintomas de curto prazo, não é chocante o comportamento de empresários em evitá-las. No entanto, uma compreensão mais ampla, num espectro com relevância empresarial, associa as tendências com as mudanças relacionadas ao mercado. A previsão de tendências consiste principalmente na identificação de grandes comportamentos e tendências sociais e adaptá-las em direcionamento criativo para produtos e serviços. Essas duas diferentes compreensões de tendência refletem as duas principais tipologias de tendências: tendências socioculturais amplas e tendências orientadas para produtos.

\section{Terminologias para tendências}

A conotação contemporânea de tendência abre um leque de sinônimos, especificações e terminologias dentro do escopo semântico da palavra. Moda, macrotendência, microtendência, megatendência, onda, modinha, gimmick, fad, craze e tendência de curto, médio e longo prazo, etc.. A variedade de termos elucida que em estudos científicos e no mercado profissional há pouca coerência no uso de terminologias. Isso leva à confusão e compromete a concordância dentro do setor e entre profissionais e acadêmicos. Além disso, os parâmetros para a classificação das tendências são, às vezes, arbitrários e vinculam aspectos não diretamente proporcionais; por exemplo, rapidez e amplitude de difusão (ERNER, 2012).

O Quadro 1 foi desenvolvido com o propósito de sistematizar as diferentes categorias utilizadas como referência às tendências. A organização dos dados ainda promove a identificação de convergências e equivalências dentro da gama de expressões. Organizou-se o quadro de modo a apresentar, na primeira coluna o a publicação, o autor e o ano da publicação. A segunda coluna, desenvolvida pelos autores, exibe os parâmetros considerados para a definição das categorias, essas por sua vez listadas na terceira coluna. As diferentes categorias estão listadas ordenadamente, sendo que aquelas apresentadas inicialmente são menos impactantes e duradouras do que aquelas apresentadas ao fim. 


\begin{tabular}{|c|c|c|}
\hline autor, ano e publicação & parâmetro & categorias \\
\hline $\begin{array}{l}\text { Erner, G.(2012) } \\
\text { Sociologia das tendências }\end{array}$ & $\begin{array}{l}\text { amplitude de adoção } \\
\text { relação de pertencimento ou distinção }\end{array}$ & $\begin{array}{l}\text { tendências massivas } \\
\text { tendências confidenciais }\end{array}$ \\
\hline $\begin{array}{l}\text { Riezu, M,(2009) } \\
\text { Coolhunting }\end{array}$ & $\begin{array}{l}\text { duração (ciclo de vida) } \\
\text { potencial de impacto } \\
\text { amplitude de adoção } \\
\text { satisfação de necessidade ou desejo }\end{array}$ & $\begin{array}{l}\text { tendência } \\
\text { Fads ou hypes }\end{array}$ \\
\hline $\begin{array}{l}\text { Godart, F.(2010) } \\
\text { Sociologia da moda }\end{array}$ & $\begin{array}{l}\text { duração (ciclo de vida) } \\
\text { amplitude de adoção } \\
\text { difusão }\end{array}$ & $\begin{array}{l}\text { modas } \\
\text { entusiasmos }\end{array}$ \\
\hline $\begin{array}{l}\text { Kotler, P. \& Keller, K.(2006) } \\
\text { Marketing Management }\end{array}$ & $\begin{array}{l}\text { duração (ciclo de vida) } \\
\text { potencial de impacto } \\
\text { previsibilidade } \\
\text { base sociocultural }\end{array}$ & $\begin{array}{l}\text { tendência sociocultural } \\
\text { moda } \\
\text { onda }\end{array}$ \\
\hline $\begin{array}{l}\text { Solomon, M. (2008) } \\
\text { Consumer Behavior }\end{array}$ & $\begin{array}{l}\text { duração (ciclo de vida) } \\
\text { potencial de impacto }\end{array}$ & $\begin{array}{l}\text { clássico } \\
\text { moda } \\
\text { modinha }\end{array}$ \\
\hline $\begin{array}{l}\text { Buck, Hermann \& Lubkowitz (1998) } \\
\text { Handbuch Trend-management }\end{array}$ & $\begin{array}{l}\text { duração (ciclo de vida) } \\
\text { potencial de impacto }\end{array}$ & $\begin{array}{l}\text { clássico } \\
\text { tend. de longo prazo } \\
\text { tend. de curto prazo } \\
\text { fenômeno de moda }\end{array}$ \\
\hline $\begin{array}{l}\text { McCracken, G.(2011). } \\
\text { Chief Culture Officer }\end{array}$ & $\begin{array}{l}\text { duração (ciclo de vida) } \\
\text { potencial de impacto } \\
\text { amplitude de adoção } \\
\text { base sociocultural }\end{array}$ & $\begin{array}{l}\text { cultura lenta } \\
\text { cultura rápida }\end{array}$ \\
\hline $\begin{array}{l}\text { Caldas, D. (2004) } \\
\text { Observatório de sinais }\end{array}$ & $\begin{array}{l}\text { duração (ciclo de vida) } \\
\text { base sociocultural }\end{array}$ & $\begin{array}{l}\text { tendências de fundo } \\
\text { tendências de curto prazo }\end{array}$ \\
\hline $\begin{array}{l}\text { Lipovetsky, G. (2012) } \\
\text { Qu'est-ce qu'une tendance? }\end{array}$ & $\begin{array}{l}\text { base sociocultural } \\
\text { arbitrariedade }\end{array}$ & $\begin{array}{l}\text { tendências pesadas } \\
\text { tendências leves }\end{array}$ \\
\hline $\begin{array}{l}\text { Baldini, } M,(2006) \\
\text { The invention of fashion }\end{array}$ & $\begin{array}{l}\text { duração (ciclo de vida) } \\
\text { potencial de impacto } \\
\text { amplitude de adoção }\end{array}$ & $\begin{array}{l}\text { traje } \\
\text { moda } \\
\text { fad } \\
\text { craze }\end{array}$ \\
\hline $\begin{array}{l}\text { Naisbitt, J.; Aburdene, P.(1991) } \\
\text { Megatrends } 2000\end{array}$ & $\begin{array}{l}\text { potencial de impacto } \\
\text { base sociocultural }\end{array}$ & megatendência \\
\hline $\begin{array}{l}\text { Rehn, A. \& Lindkvist, M.(2013) } \\
\text { Trendspotting }\end{array}$ & $\begin{array}{l}\text { duração (ciclo de vida) } \\
\text { potencial de impacto }\end{array}$ & $\begin{array}{l}\text { microtendência } \\
\text { macrotendência } \\
\text { megatendência } \\
\text { gigatendência }\end{array}$ \\
\hline $\begin{array}{l}\text { Hill, S. }(2003) \\
60 \text { trends in } 60 \text { minutes }\end{array}$ & $\begin{array}{l}\text { duração (ciclo de vida) } \\
\text { potencial de impacto }\end{array}$ & $\begin{array}{l}\text { história } \\
\text { moda } \\
\text { modinha }\end{array}$ \\
\hline
\end{tabular}


Guillaume Erner (2012) apresenta na obra totalmente dedicada às tendências a distinção entre tendências confidenciais e tendências massivas. As confidenciais são pontuais e definem pertencimento a um grupo específico. Já as massivas são de interesse de um grande número de indivíduos. Para ele, compreender a articulação entre as tendências confidenciais e massivas é complexo porque o primeiro tipo investe na distinção de indivíduos e grupos, ao passo que o segundo possibilita a integração no corpo social. Todavia a diferenciação entre os dois tipos pode desaparecer, pois uma tendência massiva é sempre uma confidencial que exultou.

No campo do coolhunting, a pesquisadora de tendências Marta Riezu (2009) também apresenta duas categorias para tendências. Pode-se distinguir uma tendência de uma fad - termo em inglês que denomina novidade, furor, capricho. No contexto de mercado, uma tendência corresponde a um elemento com duração de cerca de cinco anos, sendo possível que essa tendência sofra alterações para que se mantenha válida durante o período. Já fads têm um ciclo de vida mais curto. Sendo amplamente voláteis, afetam a um grupo limitado de consumidores e têm um pequeno potencial para alcançar impacto substancial.

Por conseguinte, para apreender a distinção entre os dois tipos de tendências é necessário fazer as seguintes questões: [1] "quem está por trás dessa tendência?", que se refere ao fator de amplitude; [2] "quais são as possibilidades de essa tendência tornar-se popular?", referenciando seu potencial de impacto; e [3] "ela aparece em mais de um lugar?", considerando novamente a amplitude da adoção, desta vez pela indústria (RIEZU, 2009). A autora elencou outro fator de diferenciação entre as categorias: se satisfaz uma necessidade ou um capricho. A conceituação ignora a categoria desejo, que parece muito mais interessante para explicar as frequentes mudanças da Moda (MEINHOLD, 2013).

Godart (2010) também apresenta apenas duas categorias de tendências: modas e entusiasmos. As modas corresponderiam a mudanças mais estruturadas e duráveis, espalhadas pela sociedade através dos distintos mecanismos sociais de difusão. Já os entusiasmos são correspondentes às fads - também empregado por Riezu - que se refere a um fenômeno de popularidade súbita e de curta duração. Enquanto a lógica das modas baseia-se em mecanismos sociais específicos, a lógica dos entusiasmos é randômica.

Kotler e Kelly (2006) fazem distinção entre onda, moda e tendência sociocultural. Os critérios de categorização respondem a duração temporal de cada tendência, sua previsibilidade e seu impacto social, econômico e político. Assim, uma onda é um fenômeno imprevisível, de curtíssima duração e com impacto baixo. É característico das ondas a rápida difusão e o também rápido desaparecimento. Já uma moda tem um alcance um pouco maior, mais duradouro e consistente. Por fim, a tendência sociocultural indica um ciclo de aceitação estável e longo e causa substancial impacto social, econômico e político.

De modo semelhante à Kotler e Keller, Solomon (2008) apresenta a distinção entre modismo, moda e clássico como categorias das tendências. 
Ele resume a relação das três com o tempo no gráfico apresentado na Figura 1. A duração de um modismo equivale exatamente ao ciclo de vida de um produto, já uma moda tem uma duração quase três vezes maior, durando cerca de cinco anos. Por sua vez, um clássico afeta um grande número de pessoas por um período temporal mais extenso, gerando inclusive mudanças comportamentais e sociais.

Os pesquisadores alemães Buck, Hermann e Lubkowitz (1998) também expressam graficamente as diferentes tipologias de tendência (Figura 2). As tendências de curto prazo fenecem rapidamente. Uma tendência de longo prazo, mesmo aplicada a um produto, percorre o trajeto mais longo antes de perder seu valor de novidade. Os autores também consideram a possibilidade de uma tendência persistir por tanto tempo no mercado que venha a estabilizar-se como um novo padrão. A última curva representa uma moda, considerando a natureza cíclica das tendências da moda e dos assuntos da mídia, o movimento percorrido é sinuoso, variando entre altos e baixos, de acordo com as fases de degeneração.

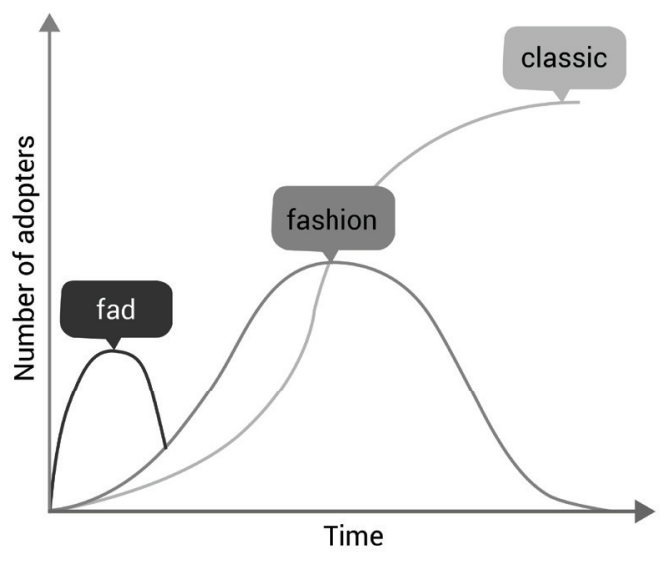

Fig 1. Comparação dos ciclos de aceitação de modismos, modas e clássicos Fonte: adaptado de Solomon, 2008, p.34

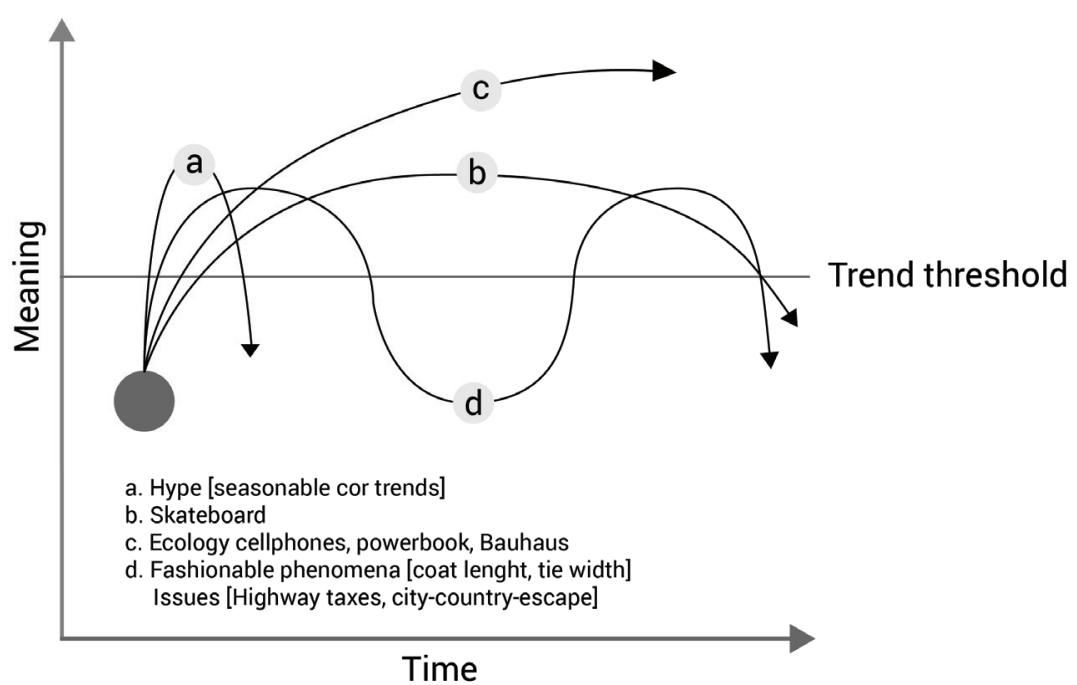

Fig 2. Desenvolvimento típico de tendências Fonte: Buck, Hermann \& Lubkotivz, 1998, p.67

O antropólogo americano Grant McCracken (2011) desenvolveu a diferenciação entre a cultura rápida e a lenta sem utilizar explicitamente a palavra tendência, todavia fortemente implícita. A sua sistematização é representativa das categorias de tendências de maior e menor impacto, e é especialmente relevante ao considerar que a cultura é um dos elementos cruciais às mudanças absorvidas pela sociedade. A cultura rápida condiz 
com as mudanças mais evidentes, perceptíveis e efêmeras. Já a cultura lenta é o pano de fundo e nunca o centro das atenções. É aquilo que está sob a superfície; menos evidente, mapeada e também mais difícil de mapear.

Divisão semelhante é defendida pelo sociólogo e pesquisador de tendências brasileiro Dario Caldas (2015). Para ele, tendências de fundo são aquelas que influenciam aspectos sociais e têm duração mais longa, enquanto tendências de curto prazo têm influência mais passageira e são coloquialmente denominadas fenômenos de moda. Normalmente, as tendências de fundo influenciam diferentes séries de tendências de curto prazo, as quais são mais voláteis e frágeis e geralmente se expressam em produtos por meio de direções estéticas e conceituais.

Gilles Lipovetsky (2012) também propõe duas categorias de tendências: leves e pesadas (em francês, tendances légères e tendances lourdes). Para o filósofo, tendências leves são movimentos que compreendem parte da arbitrariedade cultural e se inscrevem em uma lógica comercial. Elas são abruptas e ágeis, e refletem aspectos em voga e paixões. Já uma tendência pesada consiste em uma mudança real com significado social, coerência e racionalidade. São mais duradouras nas esferas social e econômica; por exemplo: o aumento da idade do casamento.

Especificamente focado no setor do vestuário, Massimo Baldini (2005) diferencia quatro categorias. O traje é qualquer fenômeno que não sofre modificações. Corresponde à norma, aos costumes, às tradições. A segunda categoria é a de moda, estilo predominante por determinada época, limitado a um período de tempo. Um fad equivale a uma epidemia mental ou moda passageira com duração de poucas semanas e atinge um número limitado de pessoas. Já o craze, que se assemelha ao fad pela rapidez e limitada abrangência, caracteriza-se pelo exagero e excentricidade.

Há também discussões que concernem a diferenciação entre as tendências socioculturais e de comportamento e as tendências de moda, já interpretadas para o nicho de mercado específico. Esse contraste influencia até mesmo o nome dado à prática da pesquisa, bem como suas técnicas. Rech e Silveira (2017) elencaram seis modalidades de estudos de tendência (trend forecasting; trend research, fashion research, fashion forecasting, lifestyle studies e future studies). As professoras apresentam que fashion forecasting "envolve a coleta de informações como estilos, cores, formas e materiais utilizados como inputs para o desenvolvimento de coleções de moda" (RECH; SILVEIRA, 2017, p.10). Já future studies compreendem abordagens e métodos amplos aplicados à busca sistematizada e planejada do futuro (Ibid, p.12).

A revisão, sistematização e interpretação da literatura direcionou para a constatação de duas amplas categorias de tendências, uma de maior e outra de menor impacto. Assim, os termos macrotendência e microtendência, foram selecionados para exibir, de forma mais definida e específica, qualidades dessas duas amplas formas de tendência. Sendo assim, os próximos itens darão ênfase às microtendências e macrotendências; bem como conceitos satélite, tais quais mega, gigatendência serão brevemente descritos. 


\section{Macrotendências}

Macrotendências têm duração de uma a duas décadas e abarcam mudanças econômicas, políticas e tecnológicas. Elas são altamente impactantes em diferentes setores e relevantes para a construção de novos cenários. As macrotendências são consideradas movimentos ou mudanças socioculturais que influenciam a sociedade por longos períodos de tempo. Seu principal parâmetro é o potencial de impacto, uma vez que alcançam repercussão no estilo de vida da sociedade, principalmente de consumidores, orientando suas preferências e gostos (BRANNON, 2010).

A pesquisa e o direcionamento de macrotendências são considerados essencialmente estratégicos para as mais diversas áreas (MOZOTA, 2010; GIMENO, 2000; MCCRACKEN, 2011; BUCK, HERMANN, LUBKOWITZ, 1998, et.al.). Macrotendências são eficazes para o planejamento estratégico a longo prazo e para o desenvolvimento de cenários. Dario Caldas (2007) declarou que as macrotendências são estratégicas na identificação de novos espaços e oportunidades para empresas, pois reverberam correntes socioculturais e possuem impacto mais duradouro na cultura, consumo e comportamento.

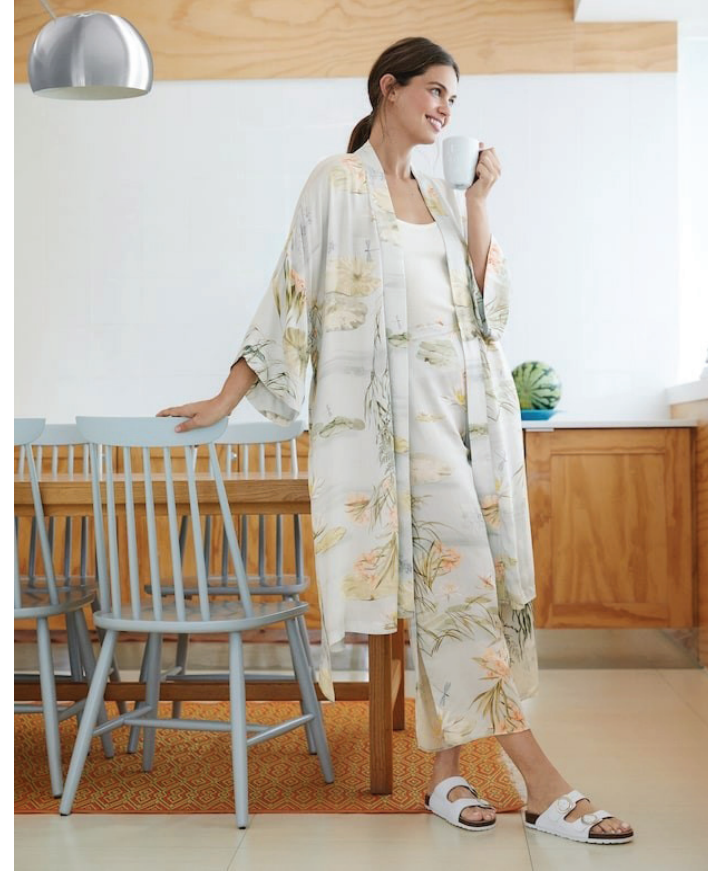

Fig 3. Imagem do lookbook Oysho Fonte: Oysho, 2020

Por exemplo, a marca Oysho (figura 3) atende ao segmento loungewear; literalmente moda para casa. A aposta do posicionamento da marca atende diretamente à macrotendência cocooning (encapsulamento) proposta por Faith Popcorn já em 1999. A tendência expressa o desejo dos indivíduos de permanecer em casa e socializar digitalmente e não pessoalmente. A tendência cresceu com o aumento da internet e tecnologias que permitem que as pessoas minimizem a saída da casa; como serviços de entrega (delivery), escritório em casa (home office) e centros particulares de entretenimento (home theater), etc.

Devido ao fato de serem transformações profundas e de alto impacto, é fundamental para o estudo das macrotendências investigar as unidades de comportamento e conduta social; principalmente nas áreas sociopolíticas, culturais, de estilo de vida, além dos campos da arte, economia, arquitetura, design e gastronomia. Todos esses diferentes setores são de interesse dos pesquisadores de tendência, já que o parâmetro de avaliação da relevância de uma macrotendência é dado pelo impacto exercido nos fluxos e estruturas da sociedade (ZANETTINI, 2012).

Além das macrotendências, há quem aplique o termo megatendência. Naisbitt \& Aburdene (1991) sugerem que elas são amplas, nem sempre evidentes e costumam durar duas décadas; o que corrobora com as características das macrotendências. Por sua vez, Lindkvist (2010) apresenta as megatendências e gigatendências como categorias adicionais à categorização dual. "Quando uma macrotendência de um segmento de uma indústria começa a afetar toda a indústria, ela se torna uma megatendência, e quando ela abrange várias indústrias e outros aspectos da sociedade, é uma gigatendência" (REHN \& LINDKVIST, 2013, p. 13-14). 


\section{Microtendências}

Por sua vez, microtendências são fenômenos de duração temporal inferior quando comparadas às macrotendências, também alcançando inferior abrangência e amplitude. Elas são altamente representativas da volatilidade contemporânea, representam influências em menor escala e, igualmente, força de intensidade inferior (ZANETTINI, 2012). Observáveis, relacionam-se a mudanças pequenas e perceptíveis que ocorrem cotidianamente ao nosso redor: o que vestimos, o que preferimos comer, sobre quais assuntos preferimos conversar, até mesmo quais palavras utilizamos para expressar-nos.

Um exemplo de microtendência é a chamada athleisure. Tal tendência corresponde ao uso de roupas esportivas como roupas de lazer regular, devido ao seu conforto e à indicação de um estilo de vida saudável e relaxado. A athleisure combinaria as exigências do estilo de vida rápido, ao aderir funcionalidade e estilo. A microtendência da moda também está relacionada à tendência fitness, notável desde o ano de 2010, mas que se mostra de forma mais incisiva desde 2013. A tendência foi benéfica, evidentemente, para marcas de roupas esportivas (sportswear), como Adidas, Nike e Puma, que se associaram com renomados estilistas e designers para aprimorarem a oferta de produtos das marcas (Figura 4).

Muitos pesquisadores utilizam microtendências para identificar macrotendências mais amplas e substanciais, o que sugere que há macrotendências por detrás das microtendências, atuando em segundo plano. Pode-se concluir que as microtendências são derivações de impacto mais baixo de tendências de fundo - mais firmemente baseadas em forças sociais e culturais - que influenciam mais diretamente produtos e serviços. As microtendências geralmente são apresentadas como direção do produto. Buck, Hermann e Lubkowitz (1991) consideram que uma tendência de produto é uma tendência dirigida à solução do produto, fundamentada não só em mudanças tecnológicas, mas também em culturais e estéticas, que têm como base os desenvolvimentos do meio onde o produto é desenvolvido e comercializado.

Apesar de fácil de aplicar, este tipo de tendência pode ser perigoso. De acordo com McCracken (2011), elas podem ser uma benção e uma maldição. Ao mesmo tempo em que elas abrem oportunidades fascinantes, são extremamente traiçoeiras porque qualquer mudança no vetor pode causar uma reviravolta que altera completamente o cenário. Muitos preferem não pesquisar microtendências. Há outros que as consideram deveras importantes, pois apresentam indícios notáveis sobre o futuro. Aqueles favoráveis à investigação das microtendências usufruem de seu conteúdo ao extrapolar seus limites para obter um vislumbre mais amplo de eventos (REHN \& LINDKVIST, op. cit.). Sendo assim, essa categoria pode fornecer o primeiro passo de um processo longo e profundo de pesquisa prospectiva. 


\section{Discussões}

Como contribuição final desta pesquisa de revisão e atualização da bibliografia sobre tendências, desenvolveu-se dois quadros resumo dos resultados. 0 quadro 2 apresenta as duas categorias de tendências, diferenciando-as a partir dos principais parâmetros de sistematização de tendências levantados na pesquisa.

\begin{tabular}{l|l|l}
\hline & macrotendência & microtendência \\
\hline adoção & ampla & restrita \\
\hline pertencimento & geral & particular \\
\hline duração & longa & breve \\
\hline impacto & substancial & sutil \\
\hline difusão & mais lentamente & mais velozmente \\
\hline previsibilidade & difícil & fácil \\
\hline base sociocultural & evidente & oculta \\
\hline
\end{tabular}

Quadro 2. Sistematização dos atributos das macrotendências e microtendências a partir dos parâmetros de diferenciação. Fonte: $\mathrm{O}$ autor, 2020

Outros dois quadros possibilitam a visualização das correspondências de todos os termos levantados na investigação à dupla elegida de termos: macrotendências (Quadro 3) e microtendências (Quadro 4).

\begin{tabular}{l|l}
\hline categorias semelhantes à de macrotendência & autor/ano \\
\hline tendências confidenciais & Erner (2012) \\
\hline tendência & Riezu (2009) \\
\hline tendência sociocultural & Kotler \& Keller (2006) \\
\hline tendência de longo prazo & Buck, Hermann, Lubkowitz (1998) \\
\hline cultura lenta & McCracken (2011) \\
\hline tendências de fundo & Caldas (2015) \\
\hline tendências pesadas & Lipovetsky (2012) \\
\hline macrotendência & Rehn \& Lindkvist (2013) \\
\hline $\begin{array}{l}\text { fenômenos de impacto e duração superiores } \\
\text { aos das macrotendências }\end{array}$ & autor/ano \\
\hline megatendência & Naisbitt \& Aburdene (1991) \\
\hline $\begin{array}{l}\text { megatendência } \\
\text { gigatendência }\end{array}$ & Rehn \& Lindkvist (2013) \\
\hline
\end{tabular}

Quadro 3. Listagem de terminologias correspondentes às macrotendências

Fonte: O autor, 2020 


\begin{tabular}{l|l}
\hline categorias semelhantes à de microtendência & autor/ano \\
\hline tendências confidenciais & Erner (2012) \\
\hline & $\begin{array}{l}\text { Godart (2010) } \\
\text { Kotler \& Keller (2006) } \\
\text { Solomon (2008) }\end{array}$ \\
\hline moda(s) & $\begin{array}{l}\text { Buck, Hermann, Lubkowitz (1998) } \\
\text { Baldini (2005) } \\
\text { Hill (2003) }\end{array}$ \\
\hline tendência de curto prazo & Buck, Hermann, Lubkowitz (1998) \\
\hline Cultura rápida & Caldas (2015) \\
\hline tendências leves & McCracken (2011) \\
\hline microtendências & Lipovetsky (2012) \\
\hline fenômenos de impacto e duração inferiores & Rehn \& Lindkvist (2013) \\
\hline aos das microtendências & autor/ano \\
\hline & Riezu (2009) \\
\hline fads, hypes, craze & Solomon (2008) \\
\hline entusiasmos & Baldini (2005) \\
\hline & Kill (2003) \\
\hline Godart (2010)
\end{tabular}

Quadro 4. Listagem de terminologias correspondentes às microtendências Fonte: O autor, 2020

Uma macrotendência consiste em mudança com efeito duradouro, estendendo-se de cinco a vinte anos. Elas são bem fundamentadas por mudanças em aspectos culturais, sociais, políticos e econômicos e influenciam diferentes áreas da vida social. Macrotendências são largamente adotadas, influenciando a população em geral. Devido a tais características, pode-se afirmar que macrotendências têm relação com os fenômenos intitulados como tendências massivas, socioculturais, pesadas, de fundo, de longa duração e cultura lenta (ERNER, 2012; RIEZO, 2009; KOTLER, LIPOVETSKY, 2012; KELLER, 2006; BUCK, HERMANN, LUBKOWITZ, 1998; MCCRACKEN, 2011;CALDAS, 2004). Expressões ainda mais duradoras de macrotendências são denominadas megatendências ou gigatendências.

Por sua vez, as microtendências geralmente têm menor resistência e impacto. Com duração de até cinco anos, elas podem ser consideradas como a aplicação de macrotendências em áreas específicas, geralmente relacionadas a aspectos estéticos. Por serem relegadas à aparência e aos atributos expressivos dos objetos e não explicitarem sua relação direta com 
as tendências socioculturais e são consideradas arbitrárias. Mesmo tendo difusão viral, raramente abrangem a sociedade de modo completo e fundamental. Outros termos recorrentes são tendências confidenciais, de curto prazo, cultura rápida, tendências leves e moda (Ibid.). Os termos entusiasmo, onda, modinha fad, craze, hype, correspondem a fenômenos de duração e impacto ainda menos intensos. Todavia, situações endêmicas podem tornar-se epidêmicas, ampliando sua abrangência e duração.

\section{Considerações}

Aparentemente consubstanciais à ideia de moda, as tendências influenciam a vida social de modo direto. Da preferência por certas teorias científicas à estrutura das famílias, da nossa relação com os alimentos à nossa relação com os utensílios. Os especialistas da área pedem urgência e seriedade no estudo das e sobre as tendências. $O$ uso desregrado e incerto do termo dificulta sua compreensão, bem como sua aplicação; uma vez que tendências podem ser empregadas de modo estratégico, auxiliando a tomada de decisões.

Este artigo teve como objetivo problematizar teórica e conceitualmente as tendências, principalmente suas taxonomias e categorizações. Dentro de uma gama numerosa e nebulosa de tipologias, propôs-se a utilização dos termos macrotendência e microtendência para designar as duas mais comuns categorias expressas do fenômeno. A bibliografia revisada foi composta de livros reconhecidos sobre a temática, nos quais autores propuseram alguma diferenciação entre dois ou mais tipos de tendências. Evidentemente um estudo mais amplo sobre tendências envolveria outros autores não citados aqui, devido ao fato deles não proporem uma categorização para as tendências. Como proposta para futuras pesquisas, sugere-se estabelecer parâmetros de comparação entre as terminologias aplicas a áreas específicas do design (como o design de moda, design industrial, design de interiores, design gráfico, e de branding) analisando os termos cunhados, as metodologias de pesquisa aplicadas e as plataformas de informação empregadas. 


\section{Referências}

BRANNON, Evelyn. Fashion forecasting. 3a ed. New York: Fairchild Books, 2010.

BUARQUE DE HOLANDA, Aurélio. Novo dicionário Aurélio. Rio de Janeiro: Editora Nova Fonte, 1999.

BUCK, Alex; HERMANN, Christoph; LUBKOWITZ, Dirk. Handbuch Trendmanagement: Innovation und Asthetik als Grundlage unternehmerischer Erfolge. Frankfurt: Frankfurter Allgemeine, 1998.

CALDAS, Dario. Entrevista por Juliana Dornelles em 21 de janeiro de 2007. Disponível em: <http://julianadornelles.com.br/texts/noticias_ver/id/232/>. Acesso em: 25 nov. 2010.

CALDAS, Dario. Observatório de sinais: teoria e prática da pesquisa de tendências. ed. at. São Paulo: Editora SENAC SP, 2015.

CAMPOS, Amanda Queiroz. Of tradition and creation: the discourse of fashion designers on trend report usage. 2017. Tese (Doutorado em Design) -Universidade Federal de Santa Catarina e Bergische Universität Wuppertal. Florianópolis/Wuppertal, 2017. Disponível em: <http:// elpub.bib.uni-wuppertal.de/servlets/DocumentServlet?id=7500>. Acesso em: 11 jan.2018.

CORBIN, Juliet. STRAUSS, Anselm. Basics of qualitative research: techniques and procedures for developing Grounded Theory. 3rd edition. London: Sage, 2008.

ERNER, Guillaume. Sociología de las tendencias. $1^{\underline{a}}$ ed. $2^{\underline{a}}$ reimp. Barcelona: Editorial Gustavo Gili, 2012.

FARM RIO + ADIDAS. Moletom cropped cirandeira. Disponível em <http://www.farmrio. com.br/br/produto/moletom-cropped-cirandeira/_/A-243140_7007.ptbr.farmrio> . Acesso em: 2 fev. 2017.

GIMENO, Jose Maria Ibañez. Gestion del diseño en la empresa. Madrid: McGraw-Hill/Interameticana de España, 2000.

GODART, Fréderic. Sociologia da moda. São Paulo: Editora SENAC SP, 2010.

KOTLER, Philip; KELLER, Kevin Lane. Administração de marketing. $12^{\text {a }}$ ed. São Paulo: Pearson Prentice Hall, 2006.

LEHNERT, Gertrud; KÜHL, Alicia; WEISE, Katja. (Hg.). Modetheorie: Klassische Texte aus vier Jahrhunderten. Fashion Studies. Bielefeld: transcript Verlag, 2014.

LINDKVIST, Magnus. $\mathbf{O}$ guia do caçador de tendências: como identificar as forças invisíveis que moldam os negócios, a sociedade e a vida. São Paulo: Editora Gente, 2010.

LIPOVETSKY, Gilles. Qu'est-ce qu'une tendance ? p.6-15. In: BACRIE, L.ydia (Ed.) Qu'est-ce qu'une tendance de mode? Paris: Fédération Française du Prêt à Porter Féminin, 2012.

MCCRACKEN, Grant. Chief culture officer: como a cultura pode determinar o sucesso ou fracasso de uma organização. São Paulo: Aleph, 2011.

MEINHOLD, Roman. Fashion myths: a cultural critique. Wetzlar: Transcript Verlag, 2013. MOZOTA, Brigitte Borja de. Gestão do Design: usando o design para construir valor de marca e inovação corporativa. Porto Alegre: Bookman, 2011.

NAISBITT, John; ABURDENE, Patricia. Megatrends 2000: Ten new directions for the 1990's. New York: Avon Books, 1991.

OYSHO. Coleção Classic Rosette. Disponível em: <https://www.oysho.com/pt/editorial/ classic-rossette-c1010191501.html>. Acesso em: 17 fev. 2017.

PALADINI, Edson Pacheco. Gestão estratégica da qualidade: princípios, métodos e processos. $2^{\mathrm{a}}$ ed. São Paulo: Atlas. 2009. 
PETERMANN, Elisabeth. Archeology of the Future. Reconsidering the place and nature of trend forecasting in design discourse. DRS 2014. Anais... Umea: University of Design, 2014. np. Disponível em: <http://www.drs2014.org/media/654126/0164-file1.pdf> . Acesso em: 7 set. 2015.

RECH, Sandra; SILVEIRA, Icléia. Abordagem terminológica dos estudos de tendências. $13^{\circ}$ Colóquio de Moda - 9ª Edição Internacional, 2017, Bauru, SP. Anais... Bauru, SP: UNESP/ABEPEM, 2017, p. 1-15. Disponível em: <http://www.coloquiomoda.com.br/anais/anais/13-Coloquio-de-Moda_2017/GT/gt_6/gt_6_ABORDAGEM_TERMINOLOGIA_DOS.pdf>. Acesso em: 10 jan. 2018.

REHN, Alf; LINDKVIST, Magnus. Trendspotting: the basics. Bloomington, IN: Booktango, 2013.

RIEZU, Marta Dominguez. Coolhunting: marcando tendencias en la moda. Barcelona: Parramón Arquitectura y Diseño, 2009.

SIMMEL, Georg. Filosofía de la moda. Madrid: Casimiro Libros, 2015.

SOLOMON, Michael. o Comportamento do consumidor: comprando, possuindo e sendo. $7^{\mathrm{a}}$ ed. Porto Alegre: Bookman, 2008.

SONEIRA, Abelardo Jorge. La "Teoria fundamentada em los datos" (Grounded Theory) de Glaser y Strauss. p.153-173. In: GIALDINO, Irene Vasilachis (coord.). Estrategias de investigación cualitativa. Buenos Aires: Gedisa Editoral, 2007.

VEBLEN, Thorstein. The theory of the leisure class. 2nd edition. Project Gutenberg, 2013. Disponível em: <http://www.gutenberg.org/files/833/833-h/833-h.htm\#link2HCH0006>. Acesso em: 14 jan. 2015.

ZANETTINI, Juliana. Pesquisa de tendências: uma abordagem sob a ótica do design de moda. 2012. 118f. Dissertação (Mestrado) - Programa de Pós Graduação em Design da Universidade Anhembi Morumbi, São Paulo, 2012. Disponível em: <http://www.anhembi.br/ppgdesign/ pdfs/26-juliana_zanettini.pdf>. Acesso em: 10 jun. 2014. 